\title{
Elaboration en permanence en faveur de la sécurité des patients
}

\author{
Isabelle Praplan \\ H+ Les Hôpitaux de Suisse, Cheffe de projet Qualité et sécurité des patients (département Gestion)
}

Les hôpitaux et les cliniques s'efforcent de fournir la meilleure qualité possible et d'aller au-delà des exigences légales. Ils participent à des initiatives sur une base volontaire, effectuent des mesures de la qualité qui vont plus loin que le plan de mesure de l'ANQ, contribuent à de nombreux registres et se font certifier. Les instruments et les organisations remplissent leur rôle. Il conviendra à l'avenir de les renforcer. $\mathrm{H}+$ soutient ses membres et encourage les approches pour la sécurité des patients.

La sécurité des patients dans les hôpitaux de soins aigus et psychiatriques ainsi que dans les cliniques de réadaptation repose sur de nombreux éléments. Elle commence dès avant l'admission du patient dans une institution et se poursuit après sa sortie: pour les hôpitaux et les cliniques, il est naturel de garantir la sécurité des patients à toutes les étapes du processus.

\section{Bases légales}

La loi fédérale sur l'assurance-maladie (LAMal) prévoit que les hôpitaux et les cliniques doivent prodiguer les prestations fixées par la loi et figurant sur la liste hospitalière de manière efficiente et dans la qualité nécessaire. A cet effet, $\mathrm{H}+$ a développé la solution de branche Qualité. Celle-ci prévoit un modèle uniforme pour les rapports sur la qualité des hôpitaux, la promotion systématique de directives sur la qualité et d'activités d'amélioration. Elle comprend aussi le portail d'information www.info-hopitaux. ch. H+ est par ailleurs un des partenaires de Sécurité des patients Suisse et de l'Association nationale pour le développement de la qualité dans les hôpitaux et les cliniques (ANQ), ce qui fait d'elle un acteur important du réseau national de la qualité.

\section{Les Peer Reviews interprofessionnels, un nouveau projet national}

Avec la Fédération des médecins suisses (FMH) et l'Association suisse des directrices et directeurs des services infirmiers (ASDSI), H+ promeut les Peer Reviews interprofessionnels. L'efficacité des Peer Reviews est démontrée. Cette démarche consiste à analyser des dossiers de patients avec le concours de collègues externes lorsque des anomalies statistiques sont identifiées et, au besoin, à élaborer des mesures d'amélioration. Des Peer Reviews sont déjà régulièrement mis en œuvre dans de nombreux hôpitaux suisses.

La nouveauté du projet en cours consiste à développer au niveau national des Peer Reviews interprofessionnels en collaboration avec l'association allemande Initiative Médecine de qualité (Initiative Qualitätsmedizin IQM). Il s'agit d'adapter aux particularités suisses la procédure de l'IQM reconnue au niveau international et de la tester dans des projets pilotes. Les documents sont mis à disposition dans les trois langues officielles et les soins infirmiers sont intégrés, ce qui constitue

Les résultats des mesures de la qualité et les activités en faveur de la sécurité des patients peuvent être consultés sur le portail infohopitaux.ch pour chacune des institutions.

une innovation pour l'IQM. Grâce à l'approche interprofessionnelle, les cas critiques peuvent être pris en considération dans leur globalité et des mesures d'amélioration de la sécurité des patients peuvent être élaborées et mises en pratique de concert.

\section{info-hopitaux.ch - le portail de recherche d'hôpitaux}

Grâce à ses diverses fonctions de recherche, le portail info-hopitaux.ch de $\mathrm{H}+$ permet de trouver de manière ciblée un hôpital qui correspond aux besoins de chacun. Ce site apporte non seulement une aide aux patients et à leurs proches pour la recherche d'un hôpi- 
H+ Les Hôpitaux de Suisse est l'association nationale des hôpitaux, cliniques et institutions de soins publics et privés. Elle compte 236 hôpitaux, cliniques et établissements médico-sociaux - répartis sur 369 sites - en tant que membres actifs et près de 170 associations, administrations, institutions, entreprises et particuliers comme membres partenaires. A travers ses institutions membres, $\mathrm{H}+$ représente quelque 185000 personnes actives.

tal mais aussi une contribution déterminante à la transparence de la branche hospitalière dans le domaine de la qualité.

Lors d'une intervention planifiée, les patients ou leurs proches ont souvent le choix entre plusieurs hôpitaux et cliniques. Il n'est pas toujours facile de se décider en faveur de tel ou tel fournisseur de prestations. Infohopitaux.ch apporte une assistance. Les fonctions de recherche par lieu et par palette de prestations facilitent le choix et fournissent des informations qui viennent compléter la recommandation du médecin de famille ou du spécialiste. Tous les membres actifs de $\mathrm{H}+$ des domaines des soins somatiques aigus, de la psychiatrie et de la réadaptation sont présents sur le portail. Une recherche par services des urgences est également disponible, avec possibilité de distinguer entre les urgences médicales, pédiatriques, gynécologiques ou psychiatriques.

\section{Transparent, fiable et complet}

Les contenus du portail se basent sur les statistiques de prestations de l'Office fédéral de la statistique (OFS) validées, ainsi que sur les informations fournies par les hôpitaux et les cliniques, parmi lesquelles figurent les rapports sur la qualité ainsi que les rapports annuels. En outre, les institutions peuvent compléter leur profil avec d'autres données et l'agencer de manière attrayante. Les résultats des mesures de la qualité et les activités en faveur de la sécurité des patients peuvent aussi être consultés sur le portail pour chacune des institutions. H+ exploite le site et veille à l'uniformité des présentations.

Outre le site web, une application mobile «info-hopitaux" est disponible pour les smartphones. Elle se base sur les mêmes informations que le portail mais elle est destinée avant tout aux urgences.

Correspondance: Isabelle Praplan H+ Les Hôpitaux de Suisse Lorrainestrasse $4 \mathrm{~A}$

$\mathrm{CH}-3013$ Berne

Tél. 031335111

\section{Solution de branche de $\mathrm{H}+$}

H+ Les Hôpitaux de Suisse met à la disposition de ses membres un modèle pour leurs rapports sur la qualité afin d'offrir au public intéressé, aux patients et aux spécialistes de la santé un aperçu systématique aussi clair que possible des activités menées par un hôpital ou une clinique en faveur de la qualité. Les institutions peuvent publier leurs rapports sur le portail info-hopitaux.ch, une possibilité exploitée par 182 sites d'hôpitaux en 2014. La plupart des hôpitaux ont utilisé le modèle de rapport de $\mathrm{H}+$.

\section{Sécurité des patients Suisse}

La Fondation pour la Sécurité des patients Suisse est organisée en réseau. Elle analyse les problèmes de sécurité et développe, propage et évalue des solutions pour les fournisseurs de prestations. Elle encourage l'implantation de ces solutions. Toutes ses activités ont pour but de contribuer à améliorer la sécurité des patients et à prévenir les erreurs dans la fourniture des soins.

Dans le domaine de la chirurgie, la Fondation pour la Sécurité des patients Suisse a développé des directives en collaboration avec des experts et des sociétés de discipline de la médecine invasive. L'élément de sécurité central est une check-list utilisée en salle d'opération. L'efficacité des check-lists en chirurgie a été démontrée par des études internationales. Ces check-lists permettent de réduire la fréquence des décès, des complications et des erreurs. Leur succès dépend du soin apporté à leur introduction et à leur application dans les institutions. Le programme propose une aide et un appui. L'objectif est d'aboutir à une utilisation généralisée et méthodologiquement correcte des check-lists et, par voie de conséquence, à une réduction des incidents et des erreurs. La culture de la sécurité et l'esprit d'équipe dans les organisations de santé en sortent ainsi renforcés. Le programme "progress! La sécurité en chirurgie» a démarré à l'été 2013.

\section{Plus de 70 registres médicaux}

Un registre médical peut être défini comme un recensement de données médicales et/ou économiques relatives à la population ou aux patients. Les registres médicaux sont, traditionnellement, des instruments de la recherche épidémiologique. Mais ils servent toujours plus souvent à l'assurance de la qualité et à la recherche sur l'offre en soins. Ils apportent ainsi une contribution essentielle à la sécurité des patients. Actuellement, plus de 70 registres médicaux enregistrent en Suisse des données sur les maladies et sur la qualité. 